\title{
Correction to: Diagnostic impact of ascites cytology in 941 patients: malignancy rates and time of detection in ovarian cancer relative to other tumor types
}

\author{
Jens Krugmann ${ }^{1}$. Corinna Lang Schwarz ${ }^{1}$ - Balint Melcher ${ }^{1}$. William Sterlacci ${ }^{1}$. Michael Vieth ${ }^{1}$ - Sophia Rösch ${ }^{2}$. \\ Johannes Lermann ${ }^{3}$
}

Published online: 30 May 2020

(c) Springer-Verlag GmbH Germany, part of Springer Nature 2020

\section{Correction to: \\ Archives of Gynecology and Obstetrics \\ (2020) 301:1521-1532 \\ https://doi.org/10.1007/s00404-020-05553-y}

Unfortunately we did not explain the type of sample collection in "materials and methods" more exactly. The samples includes intraoperative peritoneal washing cytology and cytologies from ascites punctures. In our study we included 941 patients. Intraoperative peritoneal washing samples were obtained in 124 of 203 ovarian carcinomas, in 2 of 2 fallopian tube carcinomas and in 4 of 15 endometrium carcinomas. Ascites puncture were performed in the other
811 patients. For the sake of simplicity we named all of the samples ascites. May be the terminus liquid intraabdominal cytology describes it better. It could be a limitation of our study.

Publisher's Note Springer Nature remains neutral with regard to jurisdictional claims in published maps and institutional affiliations.
The original article can be found online at https://doi.org/10.1007/ s00404-020-05553-y.

Jens Krugmann

jens.krugmann@klinikum-bayreuth.de

1 Institute of Pathology, Klinikum Bayreuth, Preuschwitzer Strasse 101, 95445 Bayreuth, Germany

2 Department of Obstetrics and Gynecology, Klinikum Bayreuth, Bayreuth, Germany

3 Department of Obstetrics and Gynecology, University of Erlangen, Erlangen, Germany 